\title{
Investigating Perception Time in the Far Peripheral Vision for Virtual and Augmented Reality
}

\author{
Xuetong Sun \\ University of Maryland \\ College Park, Maryland \\ xtsun@umiacs.umd.edu
}

\author{
Amitabh Varshney \\ University of Maryland \\ College Park, Maryland \\ varshney@umiacs.umd.edu
}

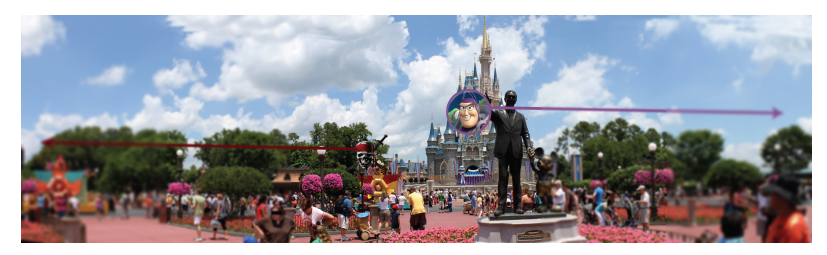

(a)

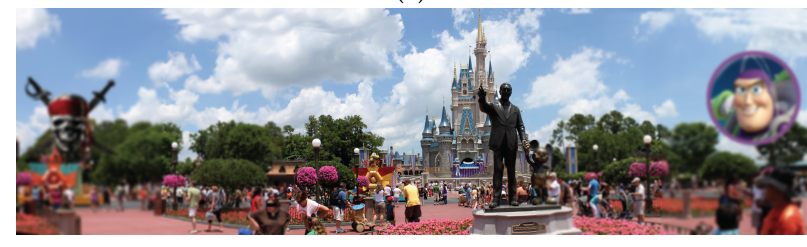

(c)

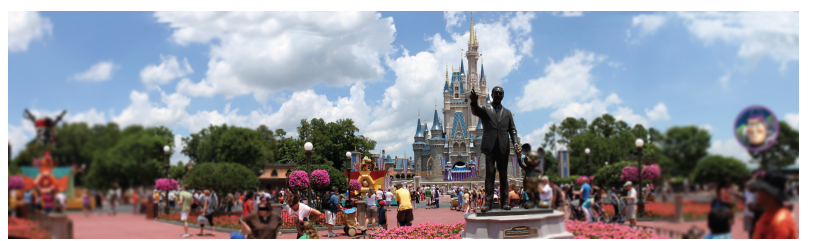

(b)

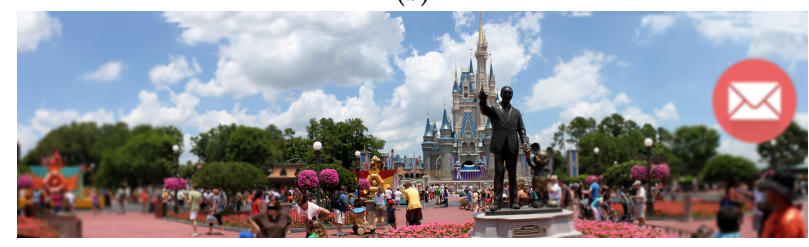

(d)

Figure 1: Understanding perception time and scale of objects in the far peripheral vision can enhance several applications in VR and AR. For example, displaying navigation information in a theme park in an AR headset should avoid (a) occluding places of interest and visual clutter of arrows, and (b) too-hard-to-notice icons. Scaling the logos and alerts according to our findings, as shown in (c) and (d) allows effective and timely perception of relevant information in the far peripheral regions without cluttering the central vision. Theme park image here is reproduced with permission from Bernie Kelm, http://www.rocket9.net.

\begin{abstract}
Far peripheral vision (beyond $60^{\circ}$ eccentricity) is beginning to be supported in the latest virtual and augmented reality (VR and AR) headsets. This benefits the VR and AR experiences by allowing a greater amount of information to be conveyed, reducing visual clutter, and enabling subtle visual attention management. However, the visual properties of the far periphery are different from those of the central vision, because of the physiological differences between the areas on the visual cortex responsible for the respective vision types. In this paper, we investigate the perception time in the far peripheral vision, specifically the time it takes for a user to perceive a pattern at a high eccentricity. We have characterized the perception time in the far peripheral vision by conducting a user study on 40 participants in which the participants distinguish between two types of patterns displayed at several sizes and at various eccentricities in their field of view. Our results show that at higher
\end{abstract}

Permission to make digital or hard copies of all or part of this work for personal or classroom use is granted without fee provided that copies are not made or distributed for profit or commercial advantage and that copies bear this notice and the full citation on the first page. Copyrights for components of this work owned by others than ACM must be honored. Abstracting with credit is permitted. To copy otherwise, or republish, to post on servers or to redistribute to lists, requires prior specific permission and/or a fee. Request permissions from permissions@acm.org.

SAP '18, August 10-11, 2018, Vancouver, BC, Canada

(C) 2018 Association for Computing Machinery.

ACM ISBN 978-1-4503-5894-1/18/08 . \$15.00

https://doi.org/10.1145/3225153.3225160 eccentricities, participants take longer to perceive a pattern. Based on user study data, we are able to characterize the desired scaling of patterns at higher eccentricities, so that they can be perceived within a similar amount of time as in the central vision.

\section{CCS CONCEPTS}

- Computing methodologies $\rightarrow$ Perception; Mixed / augmented reality; Virtual reality;

\section{KEYWORDS}

peripheral vision, perception time, virtual and augmented reality

\section{ACM Reference Format:}

Xuetong Sun and Amitabh Varshney. 2018. Investigating Perception Time in the Far Peripheral Vision for Virtual and Augmented Reality. In SAP '18: ACM Symposium on Applied Perception 2018, August 10-11, 2018, Vancouver, BC, Canada. ACM, New York, NY, USA, 8 pages. https://doi.org/10.1145/ 3225153.3225160

\section{INTRODUCTION}

Graphics has thus far primarily focused on high-fidelity rendering that is best suited for the central vision. Rendering in the far peripheral vision has not received much attention because most of the displays thus far had a narrow field of view (FOV). However, rapid advances in display technologies are enabling head mounted 


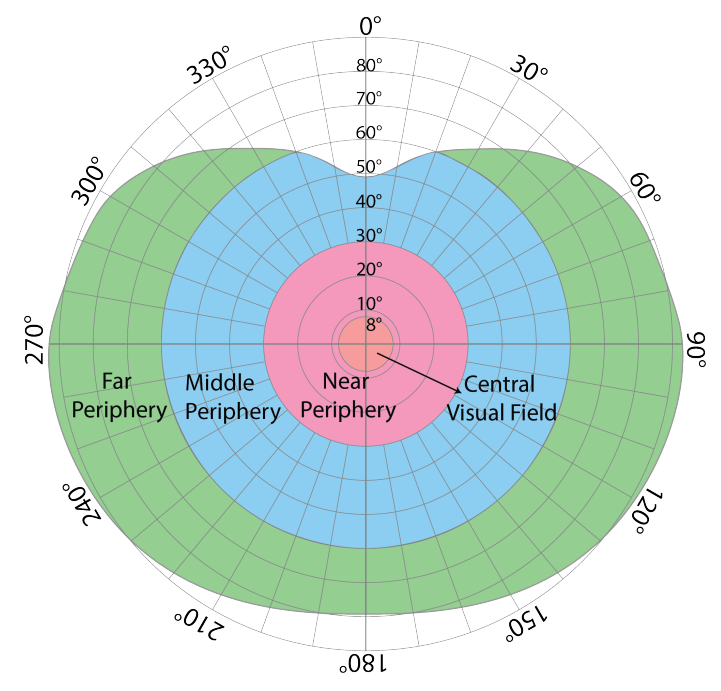

Figure 2: Illustration of human visual field. Central visual field, near, middle and far peripheral vision are from $0^{\circ}$ to $8^{\circ}, 8^{\circ}$ to $30^{\circ}, 30^{\circ}$ to $60^{\circ}$ and beyond $60^{\circ}$ respectively. Image adapted from [Jones et al. 2013b]

displays (HMDs) with an ever-increasing FOV. This has necessitated a better understanding of how peripheral vision rendering could assist in the next-generation of VR and AR applications.

In this paper, we are interested in the far peripheral vision, which suffers significantly diminished visual acuity compared to central vision. Definitions of peripheral vision differ throughout the literature. In this paper, foveal vision (within $2^{\circ}$ from the fixation point), together with perifoveal, is called the central vision [Jones et al. 2013b; Strasburger et al. 2011]. The central vision covers the region from $0^{\circ}$ to $8^{\circ}$ eccentricity, and corresponds to the eye's macula, responsible for high-resolution color vision. Beyond the central vision is the peripheral vision. Near, middle and far periphery span $8^{\circ}$ to $30^{\circ}, 30^{\circ}$ to $60^{\circ}$, and above $60^{\circ}$ eccentricity, respectively [Simpson 2017]. All eccentricities in this study are horizontal. A visual representation can be seen in Figure 2 .

Our goal in this paper is to investigate perception time in the far peripheral vision. Specifically, we conduct a user study to measure participants' perception time of a series of size-scaled simple patterns at a set of eccentricities. It takes longer to perceive a given pattern at a higher eccentricity than in the central vision; however, a larger pattern at a higher eccentricity can be perceived in a similar time as the original in central vision. Making use of the far peripheral vision has several advantages. First, peripheral vision, although lacking in visual acuity, provides an opportunity for additional information to be displayed. Second, displaying noncritical information in the far periphery can reduce visual clutter in the central vision. Last but not the least, displaying content in the far periphery is less intrusive to the central vision. Recently, Grogorick et al. [Grogorick et al. 2017] have started a very useful research direction on subtle attention management for VR and AR. Our work can be used to extend that line of research to the far peripheral vision. This allows the VR and AR users to focus on the task at hand, or fully enjoy the immersive experience while also being able to perceive information in the periphery. Displaying in the far periphery in VR and AR enables calm technology [Weiser and Brown 1996], where information, instead of overwhelming the user on a single tiny screen, is deployed and managed in a more subtle way, in this case making use of a wider field of vision. An example use case could be that when playing an immersive VR game, a message or a phone call alert could be displayed in the peripheral vision; the intrusion to the immersion is limited, yet the user is notified of new information efficiently. As another example, imagine walking in a theme park with AR headwear: directions to attractions of interest could be displayed in the far periphery, while the central view remains unobstructed. Illustrations of such use cases can be seen in Figure 1.

Our study is related to concepts in psychophysics called cortical magnification and $M$-scaling. Cortical magnification says that $M$, the diameter in the primary visual cortex onto which 1 degree of visual field projects ([Daniel and Whitteridge 1961]), is inversely linear to the eccentricity $E$. The relationship can be formulated as

$$
M^{-1}(E)=M_{0}^{-1}(1+a E)
$$

where $a$ is a coefficient [Strasburger et al. 2011]. Variations of the formula exist in [Brindley and Lewin 1968; Cowey and Rolls 1974; Horton and Hoyt 1991; Larsson and Heeger 2006; Rovamo and Virsu 1979; Schwartz 1980; Strasburger and Malania 2013; Tolhurst and Ling 1987; Tyler 1999; Yates and Stafford 2011]. This leads to M-scaling, which says performance variations with eccentricity can be minimized by using appropriately scaled stimuli ([Strasburger et al. 2011]). M-scaling can be represented as

$$
S(E)=S_{0}\left(1+E / E_{2}\right)
$$

where $S(E)$ is the scale at eccentricity $E$.

Our work makes the following contributions. First, many studies on M-scaling focus on low-level visual properties such as contrast sensitivity [Rovamo and Virsu 1979], In our study, participants are asked to perform a higher-level visual task by responding to different patterns. We record the time for participants to respond to patterns of different scales at different locations. This facilitates determination of an appropriate magnification for patterns at higher eccentricities so that they can be perceived in a similar time as those in the central visual field. Second, to our knowledge, we are the first to test and report a scaling function in the far peripheral field, applicable to a field of view of up to $140^{\circ}$ horizontally.

In this study, we investigate perception time to determine the scaling for information displayed in the far periphery that achieves a similar perception time as in the central vision. We create an automated testing system that simulates a wide field of view VR and AR display, and tracks an observer's gaze direction. In section 2 , we introduce perception studies on peripheral vision, as well as $\mathrm{VR}$ and $\mathrm{AR}$ research that makes special use of the peripheral vision. We also include previous work in designing and building wide FOV VR and AR displays. In section 3, we describe the design of our experiment, as well as our system setup. We visualize and analyze our collected data to derive the scaling function in section 4 . 
Table 1: List of wide field of view virtual and augmented reality display techniques and devices. The spatial resolution of holographic display is same as the SLM pixel pitch when inside the depth of field[Shi et al.2017]. The items with * are calculated from measurement and other reported device specifications.

\begin{tabular}{|c|c|c|c|}
\hline Display & Max reported FOV $\left({ }^{\circ}\right)$ & Color & Angular Resolution \\
\hline Spherical wave holographic display(2017) [Maimone et al. 2017; Shi et al. 2017] & $80(\mathrm{H})$ & RGB & $3.74 \mu \mathrm{m}$ \\
\hline Stereoscopic factored light field synthesis(2015) [Huang et al. 2015] & $87 \times 91$ & RGB & $4.1 \mathrm{arcmin} / \mathrm{pix}^{*}$ \\
\hline Flexible mirror membrane(2017) [Dunn et al. 2017] & $90 \times 45$ & RGB & $3.4 \mathrm{arcmin} / \mathrm{pix}^{*}$ \\
\hline LCD and optics (Meta 2) & $90 \times 50$ & RGB & $3 \mathrm{arcmin} / \mathrm{pix}$ \\
\hline Detachable display and optics (Samsung Gear VR, Google Daydream) & 100(D) & RGB & $2.5 \mathrm{arcmin} / \mathrm{pix}^{*}$ \\
\hline Pinlight(2014) [Maimone et al. 2014] & 110(D) & Mono & $4.5 \mathrm{arcmin} / \mathrm{pix}^{*}$ \\
\hline Tiling display and fresnel lenses(2003) [Massof et al. 2003] & $150 \times 100$ & RGB & $3 \mathrm{arcmin} / \mathrm{pix}$ \\
\hline LCD and catadioptical optics(2003) [Nagahara et al. 2003] & $180(\mathrm{H})$ & RGB & $5.7-10.5 \mathrm{arcmin} / \mathrm{pix}^{*}$ \\
\hline Projector and hyperbolic mirror(2007) [Kiyokawa 2007] & $190(\mathrm{H})$ & RGB & $60 \operatorname{arcmin} / \mathrm{pix}^{*}$ \\
\hline sparse peripheral display(2016) [Xiao and Benko 2016] & $190(\mathrm{H})$ & RGB & $5.5 \mathrm{arcmin} / \mathrm{pix}^{*}$ \\
\hline OLED and fresnel optics (HTC Vive, Oculus Rift, StarVR) & $210 \times 130$ & RGB & $5.5 \mathrm{arcmin} / \mathrm{pix}^{*}$ \\
\hline Curve display and optics(2016) [Rakkolainen et al. 2016a,b] & $318 \times 130$ & RGB & $5.4-7.5 \mathrm{arcmin} / \mathrm{pix}^{*}$ \\
\hline CAVE [Cruz-Neira et al. 1993, 1992; DeFanti et al. 2009; Ren et al. 2016] & full & RGB & $1.5-3 \mathrm{arcmin} / \mathrm{pix}^{*}$ \\
\hline Tiled desktop displays [Robertson et al. 1997] & full & RGB & $3-4 \operatorname{arcmin} / \mathrm{pix}^{*}$ \\
\hline
\end{tabular}

\section{RELATED WORK}

Low-level visual properties like contrast sensitivity have been studied extensively in the central and near peripheral vision. But there has been little work on far peripheral vision or on high-level perceptual functions. In section 2.1, we introduce related perceptual psychology works on peripheral vision. In section 2.2, we discuss cases in which peripheral vision is utilized in virtual and augmented reality. Last, we include a list of methods for creating wide FOV VR and AR displays in section 2.3 in Table 1.

\subsection{Studies on Peripheral Vision}

Cortical magnification and M-scaling are well-studied subjects of peripheral vision. Various models have been proposed. Cowey and Rolls [1974] first fit the visual cortex electrical stimulation data provided by Brindly and Lewin [1968] to the inverse linear model $\left(M^{-1}(E)=M_{0}^{-1}\left(E / E_{2}\right)\right)$ with $M_{0}=8.55 \mathrm{~mm} /{ }^{\circ}$ and $E_{2}=1.746$. Through medical imaging of the visual cortex, Horton and Hoyt[1991], Schira et al. [2007] and Larsson and Heeger[2006] derive different values for $M_{0}$ and $E_{2}$. Rovamo and Virsu[1979] propose adding an extra term for improved accuracy $M^{-1}(E)=\left(1+a E+b E^{3}\right) M_{0}^{-1}$ and compile four equations for the four half meridians separately. There is also the power function used by Tolhurst and Ling[1987], which is $M^{-1}(E)=M_{0}^{-1}(1+a E)^{1.1}$ with $M_{0}=24.88 \mathrm{~mm} /{ }^{\circ}$.

Rovamo and Virsu [1979] adjust the stimuli presented in the periphery using the function $M^{-1}(E)$, and the contrast sensitivity functions at different eccentricities are made similar as a result. However, it is worth noting that the cortical magnification and Mscaling concepts are not golden rules to "make everything equally visible independent of eccentricity". There are cases in which scaling does not compensate for the decreasing $M$ factor, and even cases in which visual performance is diminished [Tyler 1999; Yates and Stafford 2011]. For more details on cortical magnification and Mscaling, we refer readers to [Strasburger et al. 2011].

High-level visual functions like letter recognition are also peripheral vision study topics. Strasburger et al. [2011] propose a scaling function $S(E)=0.022 E+\frac{0.25}{\log C+2.07-0.0345 E}$ based on data from [Strasburger 2001], where $C$ is the Michelson threshold contrast. Grogoric et al.[2017] find a linear scaling function from $0^{\circ}$ to $40^{\circ}$ eccentricity to make objects presented in a virtual scene perceivable.

Studies have also shown that peripheral vision is capable of, and sometimes plays an important part in, higher-level perceptual tasks like scene and object recognition. Thorpe et al.[2001] show that even at $70^{\circ}$ eccentricity, an accuracy of $60.6 \%$ can be achieved in recognizing animal faces. Boucart et al.[2016] compare people's ability to identify images of human faces, vehicles and animals at different eccentricities. The results show that human faces have an advantage over vehicles and animals in terms of response time, and that advantage persists even at extreme eccentricities up to $80^{\circ}$. In scene gist recognition, Loschky et al. [2009; 2015] compare participants' abilities to categorize a scene from a "window" (central portion of the image) and a "scotoma" (blocking central portion, only keeping the peripheral part of the image). They show that peripheral vision is more important than central vision in scene gist recognition, as recognizing a $30^{\circ}$ radius scotoma achieves the same accuracy as recognizing the whole image, and a $5^{\circ}$ radius window is significantly harder to recognize. Other studies have shown that peripheral vision allows, though at reduced level, recognition of facial expressions and emotions ([Bayle et al. 2011; Landman et al. 2014]).

Ren et al. [2016] investigate whether a larger field of view improves searching task performance in augmented reality. Their results show that a larger field of view $\left(108^{\circ} \times 82^{\circ}\right)$ leads to shorter task completion time, but lowers correctness compared to limited field of view $\left(45^{\circ} \times 30^{\circ}\right)$. Kline and Witmer [1996] conduct a user study to investigate the effect of field of view on distance judgement in virtual environment, and find that with a smaller field of view $\left(60^{\circ} \times 38.5^{\circ}\right)$ participants tend to overestimate distance while with a larger field of view $\left(140^{\circ} \times 90^{\circ}\right)$ participants tend to underestimate. Joneset al. show in $[2017 ; 2013 \mathrm{~b}]$ that adding stimulation in the far 
periphery in a VR headset causes more accurate distance and size judgement in a virtual environment and changes people's walking behavior. A good literary review on effects of field of view in virtual environments can be found in[Jones et al. 2017].

\subsection{Peripheral Vision in VR and AR}

Researchers in the graphics community have long used the fact that human peripheral vision perceives less detail than foveal vision. Oshima et al. [1996] analyze user gaze direction, and blur objects not in the user's foveal vision to reduce rendering complexity. In an augmented reality application, Ishiguro and Rekimoto [2011] display an icon above objects in the periphery of the user's field of view, and show the detailed information when the user's gaze is moved to that object. Jones et al. [2013a] project images that are aesthetically matched with a user's content on the TV. Positive user feedback shows that enriching peripheral vision can improve the interactive user experience.

\subsection{Wide FOV Displays}

Various ways to build wide FOV VR and AR displays have been proposed. Most of the systems we review here support $100^{\circ}$ horizontal FOV or greater. The most straightforward system that extends viewer's FOV is the cave automatic virtual environment (CAVE) Cruz-Neira et al. [1993] create a $7^{\prime} \times 7^{\prime} \times 7^{\prime}$ cube-shaped CAVE where images were projected to three side walls and the floor. It covers the entire field of view when the viewer is facing the middle of the three projection walls). There have been many iterations and variations of the CAVE system [DeFanti et al. 2009; Ren et al. 2016]

In an effort to study immersion on desktop VR, Robertson et al. [1997] use two two additional monitors (referred by them as peripheral lenses) on either side of the main monitor. The total horizontal FOV using two peripheral lenses is approximately $38^{\circ}$. Attaching additional monitors can possibly cover the entire FOV.

In traditional HMDs, the two most important components are the display panel and optics. Modifying either or both is a common method of enlarging the FOV of HMDs. Nagahara et al. [2003] build a prototype video see-through HMD that uses a complicated configuration of an ellipsoidal and a hyperboloidal curved mirror to reflect light from the display into viewer's eyes, covering $180^{\circ}$ horizontal FOV. Kiyoshi [2007] create an optical see-through HMD using a hyperbolic mirror instead of a flat one to increase the horizontal FOV to $190^{\circ}$. Dunn et al. [2017] take the optics design one step further to accommodate depth cues while achieving a large field of view by using a flexible mirror membrane to reflect light from the display. This allows a $90^{\circ}$ by $45^{\circ}$ binocular FOV. The membrane is also varifocal, controlled by airtight cavities. The focal power can be adjusted with a gaze tracker. Rakkolainen et al.[2016a; 2016b] use flexible OLED that curved around viewer's eye and curved optics to reach super-wide FOV. They create proof-of-concept prototypes using fresnel lenses and achieved $232^{\circ} \times 130^{\circ}$ and $318^{\circ} \times 130^{\circ} \mathrm{FOV}$.

Tiling display panels or optics can also increase the field of view. Massof et al. [2003] achieve $150^{\circ}$ by $100^{\circ}$ FOV by using a speciallymolded array of fresnel lenses, and surrounding each lens with 16 OLED panels. Tiling optics of tiny pinholes has been used by Aksit et al. [2015]. Given the pupil and aperture size and the aperture-pupil and aperture-display distance, the FOV of a single aperture can be
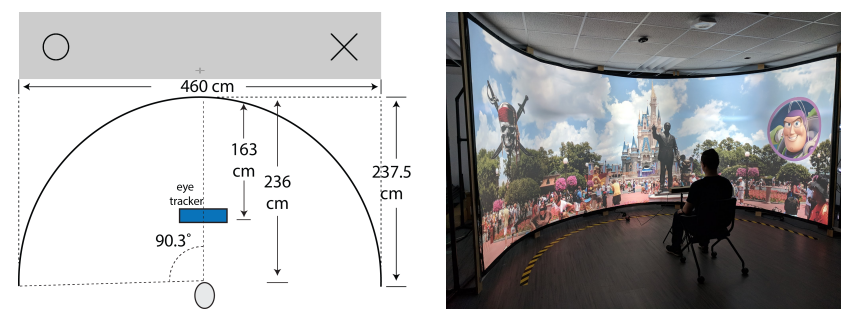

Figure 3: Top view and stimuli (left) of our user-study setup. In the experiment, patterns appear one at a time. A photograph of our system used in study (right). Shown on screen is one of the possible use cases of the system.

calculated. To widen the FOV of the HMD, an array of pinholes is used. Specially synthesized images are displayed on a cellphone, from which light travels across the apertures to reconstruct the original images. The FOV is easily expandable with larger display and more apertures. Xiao and Benko [2016] fill the periphery of the HMD with sparse arrays of high-contrast LED lights, dubbed sparse peripheral display. Their prototypes based on existing commercial VR and AR HMDs, achieve FOV of $170^{\circ}$ and $190^{\circ}$ respectively.

Light-field and holographic near-eye HMDs, while in their early development stages, have the potential to support a large FOV, and address problems like vergence and accommodation. A prototype by Huang et al. [2015] uses a rank 1-factored light field, and has a FOV of $87^{\circ}$ by $91^{\circ}$. Maimone et al.[2017] and Shi et al.[2017] use spherical light wave to address the limit of a small diffraction angle. Maimone et al.'s prototype is shown to have a $80^{\circ}$ horizontal FOV.

\section{USER STUDY DESIGN}

We build a user-study system that uses a large curved screen and eye tracking to simulate a wide FOV HMD. Stimuli of different sizes are displayed at various eccentricities. Participants respond to the stimuli by pressing buttons on a controller device. The responses are recorded. Our setup is illustrated in Figure 3.

The display we use in this study is a custom-made curved tiled screen using $5 \times 3$ projector array, with a $6400 \times 2100$ resolution. This display has a $180^{\circ}$ horizontal FOV when viewed $236 \mathrm{~cm}$ away from the center of the screen. The system setup can be seen in Figure 3 right. The eye tracking device we use is a Tobii EyeX commercial eye tracker. The eye tracker is placed $163 \mathrm{~cm}$ away from the center of the screen, slightly below eye level facing the user.

The system displays various patterns of different sizes at different eccentricities according to a pre-determined randomized order. The response from the user's game controller is recorded at $60 \mathrm{~Hz}$.

\subsection{Task and Stimuli}

We display two types of patterns, crosses and circles, as shown in Figure 3 left top. In the user study task, one type of pattern is specified as the target, and the other is the distraction. The task is to perceive the pattern and respond (by pressing a button on a controller) to the target pattern as soon as it appears and ignore the distraction, while keeping fixated at the center of the screen. For the purposes of this study, we define the time from the appearance of the pattern to the button being pressed is recorded as the perception 
time. We recognize that this includes the time for the eye to perceive the pattern, the brain to understand the pattern, and for the user to respond by pressing the button on the controller. If a participant responds incorrectly, the stimulus will turn red. For example, when the cross is specified as the target, and the circle is the distraction, the participant should press a button on the controller as soon as he or she sees a cross on the screen, and do nothing with a circle. Participants are further directed to keep looking at the center of the screen and not move their heads or gaze directions. The cross-hair at the center will turn green if eye tracker confirms the participant is looking at the center, and is black otherwise.

Since our display supports $180^{\circ} \mathrm{FOV}$ and considering the space to display the pattern at the outermost eccentricity, the stimuli are tested at eccentricities from $0^{\circ}$ to $80^{\circ}$, with a $10^{\circ}$ interval. Because we are more interested in higher eccentricities, we test stimuli at higher eccentricities at a wider scale range. Specifically, eccentricities $0^{\circ}$, $10^{\circ}, 20^{\circ}$ and $30^{\circ}$ are tested at scales $5^{\circ}, 10^{\circ}$ and $15^{\circ}$, while eccentricities $40^{\circ}, 50^{\circ}, 60^{\circ}, 70^{\circ}$ and $80^{\circ}$ are tested at scales $5^{\circ}, 8^{\circ}, 11^{\circ}, 14^{\circ}, 17^{\circ}, 20^{\circ}$, $23^{\circ}$ and $26^{\circ}$. The scale ranges are determined after our pilot study. Each eccentricity-scale combination is repeated 5 times for each pattern as target and 2 times for each pattern as distraction (14 times in total).

\subsection{User Study Procedure}

The study is conducted with one participant at a time. We first explain to the participants the purpose of this study. We then ask them to log their basic personal information, including name, age, and whether or not they have vision correction. We then explain how the study is conducted, and perform practice rounds of the study. The experimenter answers any question at this point.

To reduce participant fatigue, the entire experiment is divided into four shorter rounds between which the participants can take a break. Two rounds have the cross as the target pattern; two have the circle as the target. For example, circle is repeated 5 times for each eccentricity-scale combination and cross is repeated 2 times for each eccentricity-scale combination. These patterns are shuffled and divided into the 2 circle-target rounds. The opposite is done for the 2 cross-target rounds. The 4 rounds are the same for each participants, but half go over them in a cross1, circle1, cross 2 circle 2 order, and the other half in a circle1, cross1, circle2, cross 2 order. During each round, the experimenter stays out of the eye tracker's range. The experiment takes about 60 minutes for each participant. After all the rounds are done, each participant was compensated with $\$ 10$. During the experiment, we recorded participants' eye movements and the game controller responses. We cross-referenced this data with the stimuli to determine the precise perception time.

For the pilot user study, we had six participants. For the formal user study we recruited 40 participants (24 male and 16 female). Every participant had a normal (31 participants) or corrected-tonormal vision ( 6 had eyeglasses and 3 used contact lenses).

\section{RESULTS}

We first visualize the perception time over all eccentricity-scale combinations. Then we characterize the relation among perception time, eccentricity and scale. Finally we quantify the optimal scaling
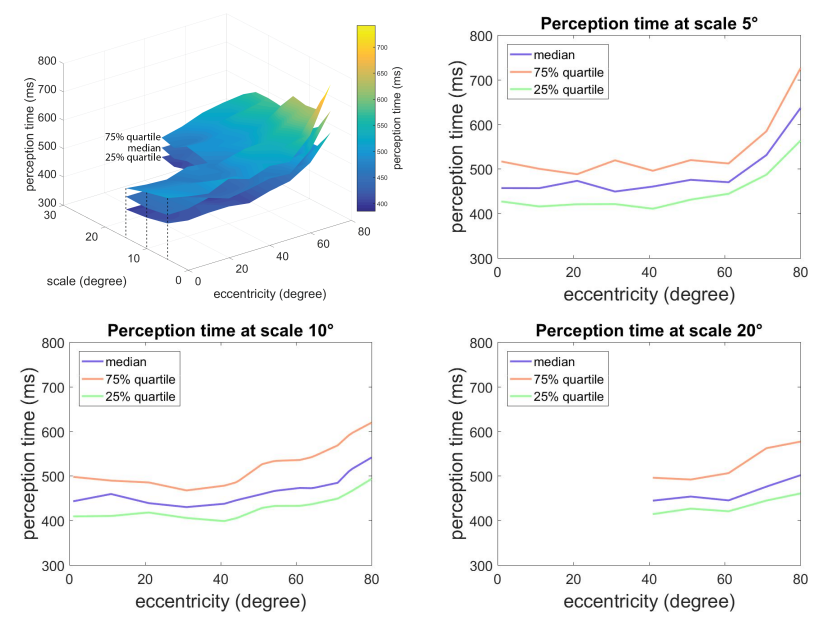

Figure 4: Perception time of all eccentricity-scale combinations is presented as a $3 \mathrm{D}$ colored mesh (top left). The three planes from top to bottom are the $75 \%$ quartile, median $(50 \%$ quartile), and $25 \%$ quartile of all participants. The perception time at three scales $\left(5^{\circ}, 10^{\circ}\right.$ and $\left.20^{\circ}\right)$ is also shown.

at high eccentricities to achieve the same perception time as in the central vision.

We first show the perception time over all eccentricity-scale combinations in the form of a 3D surface mesh. The perception time is indicated both as the height and by color. The surface mesh of the median ( $50^{\text {th }}$ percentile) as well as the $25 \%$ and $75 \%$ quartiles of all participants can be seen in Figure 4. In figure 5, the median perception time of participants over all eccentricity and scale combinations is shown in greater detail, and we plot the linear relation between scale and eccentricity that will result in the same perception time (Section 4.3). Figures 4 and 5 show that generally, perception time increases as eccentricity gets higher, and decreases as scale becomes larger. One-way ANOVA test on the perception time with each eccentricity-scale combination as a group has a $p$-value of $1.3 \times 10^{-6}$, giving us high confidence to reject the null hypothesis that the perception times follow the same distribution.

\subsection{Perception Time and Scale}

The aim of this study is to determine optimal scaling of content displayed at higher eccentricities so that it is perceived in a similar time as in the central vision. Specifically, for a pre-specified perception time $T_{0}$, we want to find at each higher eccentricity the scale at which the pattern's perception time is equal to $T_{0}$.

First we derive the relationship between perception time $T$ and scale $S$ at a given eccentricity. We plot the perception time at each tested scale for a given eccentricity directly from the user study data. We wish to represent the perception time as a function of the scale. As can be seen in Figure 6, for a given eccentricity, perception time generally decreases as scale gets larger.

Judging from Figure 6, we fit the relation of perception time and scale as a function of the form:

$$
T(S)=a \log (S)+b
$$




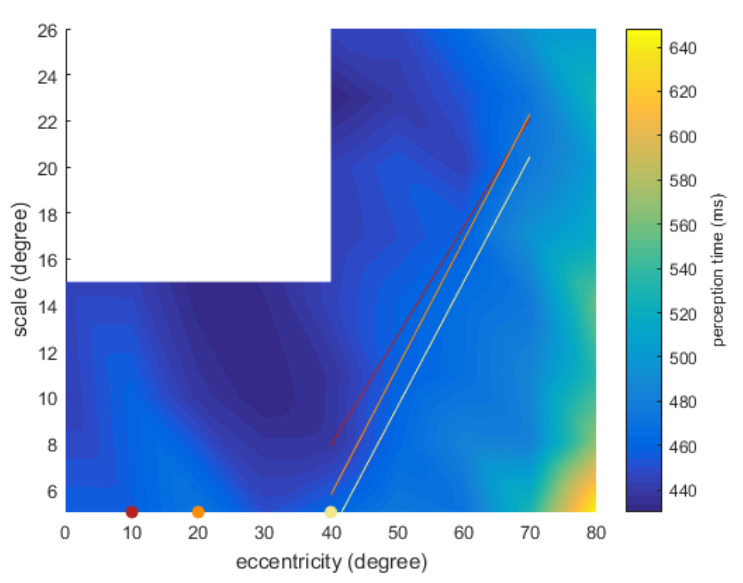

Figure 5: A color-coded map of the median perception time for all eccentricity-scale combinations. Using our findings in Section 4.3, we plot the scales at higher eccentricities to be perceived in the same time as a $5^{\circ}$ pattern at $10^{\circ}$ (red), $20^{\circ}$ (orange) and $40^{\circ}$ (yellow).

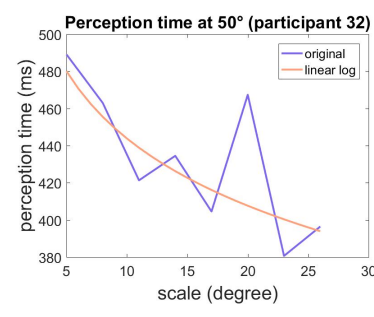

(a)

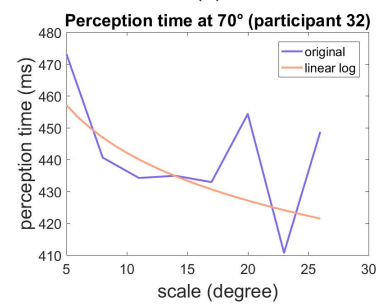

(c)

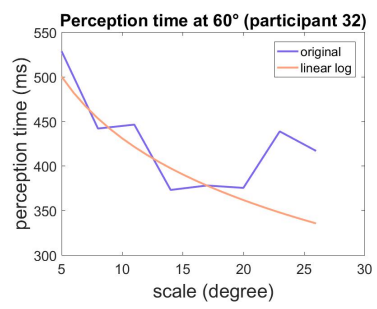

(b)

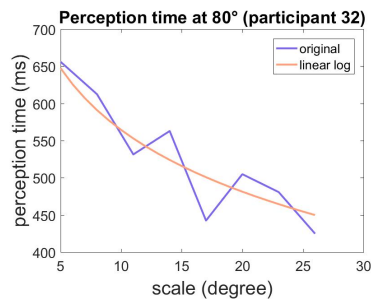

(d)
Figure 6: Figures (a), (b), (c) and (d) show the relation between perception time and scale measured for a single user (participant 32 ) at $50^{\circ}, 60^{\circ}, 70^{\circ}$ and $80^{\circ}$ eccentricities, respectively.

where $T(S)$ is the perception time in milliseconds, $S$ is the scale in degrees in the visual field, and $a, b$ are parameters. The function $T(S)$ is calculated for each eccentricity for each participant. We only calculate the perception time vs. scale relation on eccentricities above $40^{\circ}$ because we are interested in calculating this relationship for the far peripheral vision.

\subsection{Perception Time and Eccentricity}

We present the relation between perception time and eccentricity in Figure 7. Specifically, we show the median perception time over
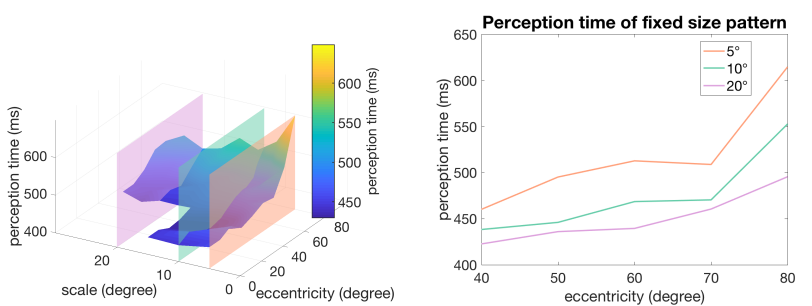

Figure 7: The perception time across eccentricities with an $s$ sized pattern is the intersection of the plane scale $=s$ and the perception time surface in Figure 4. The intersection with $5^{\circ}$, $10^{\circ}$ and $20^{\circ}$ scale planes are shown in (left). The intersection curves are shown in (right).

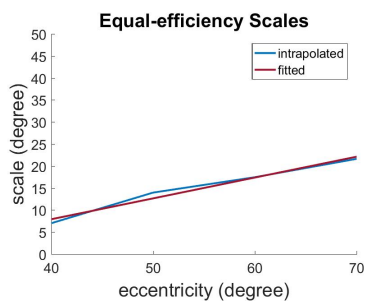

(a)

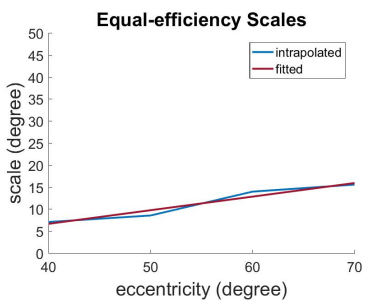

(b)
Figure 8: Equal-efficiency scales that produce the same perception time as (a) a $5^{\circ}$ pattern at $10^{\circ}$ eccentricity and (b) perceived in $450 \mathrm{~ms}$. These are the median over all participants.

all participants with $5^{\circ}, 10^{\circ}$ and $20^{\circ}$ patterns at higher eccentricities. When the size of the pattern is kept constant, it takes longer for the patterns at higher eccentricities to be perceived. Perception time decreases as the pattern size increases at the same eccentricity (see Figure 6). As is shown, there is a clear difference between the time in perceiving the same-sized pattern at a lower eccentricity versus at a higher eccentricity. The $150 \mathrm{~ms}$ difference between perceiving a $5^{\circ}$ pattern at $40^{\circ}$ and $80^{\circ}$ eccentricity is noticeable and it is meaningful to characterize the scaling at higher eccentricities to bridge this gap.

\subsection{Equal-Efficiency Scale at each Eccentricity}

With the function $T(S)$, we can calculate for any given eccentricity the scale at which the pattern is perceived within a given amount of time. Therefore, we can plot the relations of eccentricity and scale for a desired perception time. We call this the equal-efficiency scale.

Given a target perception time, we calculate the equal-efficiency scale for each participant and present the median of all participants in Figure 8. We note that we only test within the scale range of $[5,26]^{\circ}$. The equal-efficiency scales at $80^{\circ}$ eccentricity for a sizable portion of the participants are outside our tested range and therefore cannot be considered valid. The equal-efficiency scales within the tested range exhibit a linear relationship between scale and eccentricity. $S(E)=\alpha E+\beta, 40 \leq E \leq 70$.

We present the parameters for a few example target perception time choices in Table 2 . We can either use a fixed perception time, e.g. $450 \mathrm{~ms}$, or we can specify a target pattern, a pattern of a given size at 
Table 2: Parameters of the equal-efficiency scales fitted to a linear model. The valid range of eccentricity is $[40,70]^{\circ}$. Analysis of variance is conducted with the all participant's equal-efficiency scale at a certain eccentricity as a group. We include the last 5 rows because the equal-efficiency scales at $70^{\circ}$ eccentricity are no more than $4^{\circ}$ above the tested range.

\begin{tabular}{|c|c|c|c|c|c|}
\hline Target perception time & $\alpha$ & $\beta$ & $\mathrm{dF}$ & $\mathrm{F}$ & $p$-value \\
\hline $5^{\circ}$ at ecc $10^{\circ}$ & 0.47 & -11.04 & 3 & 7.02 & 0.0002 \\
\hline $5^{\circ}$ at ecc $20^{\circ}$ & 0.55 & -16.3 & 3 & 11.96 & $4.46 \times 10^{-7}$ \\
\hline $5^{\circ}$ at ecc $30^{\circ}$ & 0.54 & -15.08 & 3 & 7.96 & $5.75 \times 10^{-5}$ \\
\hline $5^{\circ}$ at ecc $40^{\circ}$ & 0.54 & -17.64 & 3 & 12.48 & $2.45 \times 10^{-7}$ \\
\hline $10^{\circ}$ at ecc $10^{\circ}$ & 0.52 & -13.11 & 3 & 4.78 & 0.0033 \\
\hline $10^{\circ}$ at ecc $20^{\circ}$ & 0.58 & -14.8 & 3 & 9.3 & $1.13 \times 10^{-5}$ \\
\hline $10^{\circ}$ at ecc $30^{\circ}$ & 0.65 & -16.52 & 3 & 7.04 & 0.0002 \\
\hline $15^{\circ}$ at ecc $10^{\circ}$ & 0.65 & -18.49 & 3 & 4.22 & 0.0067 \\
\hline $15^{\circ}$ at ecc $20^{\circ}$ & 0.51 & -6.67 & 3 & 5.32 & 0.0017 \\
\hline $15^{\circ}$ at ecc $30^{\circ}$ & 0.67 & -18.74 & 3 & 5.31 & 0.0017 \\
\hline & & & & \\
\hline
\end{tabular}

a given eccentricity. The time it takes to perceive this pattern is used as the target perception time. We also conduct one-way ANOVA tests on the equal-efficiency scales of all participants for each high eccentricity with the null hypothesis being that equal-efficiency scales over different eccentricities follow the same distribution.

We illustrate the equal-efficiency scales in a theme park navigation example in Figure 9. We choose the target perception time to be the perception time of a $5^{\circ}$ sized pattern at $10^{\circ}, 20^{\circ}$ and $40^{\circ}$, respectively. The patterns at higher eccentricities are scaled so they can be perceived within the target perception time. The target perception time increases as the eccentricity goes from $10^{\circ}$ to $40^{\circ}$. The target equal-efficiency scales decrease as a result. The scales in Figure 9 are calculated using the linear equations in Table 2.

\section{CONCLUSION AND FUTURE WORK}

Wide field of view VR and AR displays are starting to become a reality, enabling information perception in the far periphery. In this work, we conduct a user study to investigate perception time in the far peripheral vision. For a constant perception time, we found a linear relationship between eccentricity and the size of the pattern shown at that eccentricity. Based on this relation, we can optimally scale the content displayed at higher eccentricities, so that it can be perceived efficiently. This is useful for designing VR and AR experiences that are able to fully leverage the entire human visual system's field of view.

In future work, we plan to explore if the scaling function applies to even higher eccentricities. With a larger field of view display (for example StarVR ${ }^{1}$ ), we can examine the visual properties up to $105^{\circ}$ eccentricity, based on the findings in this study. In addition to visual acuity, peripheral vision also differs from central vision in several other attributes such as contrast sensitivity in color, intensity, orientation, motion, and texture detail. It will be important to investigate how these attributes affect the perception of information in the far periphery. Finally, we would like to use the findings of this study to

\footnotetext{
${ }^{1}$ https://www.starvr.com/
}

design an actual experience and show how far peripheral vision can enhance the user experience in an immersive VR or AR scenario.

\section{ACKNOWLEDGMENTS}

This project is funded by the NSF grants 14-29404, 15-64212, and the State of Maryland's MPower initiative.

\section{REFERENCES}

Kaan Akşit, Jan Kautz, and David Luebke. 2015. Slim near-eye display using pinhole aperture arrays. Applied optics 54, 11 (2015), 3422-3427.

Dimitri J Bayle, Benjamin Schoendorff, Marie-Anne Hénaff, and Pierre Krolak-Salmon. 2011. Emotional facial expression detection in the peripheral visual field. PloS one 6, 6 (2011), e21584.

Muriel Boucart, Quentin Lenoble, Justine Quettelart, Sebastien Szaffarczyk, Pascal Despretz, and Simon J Thorpe. 2016. Finding faces, animals, and vehicles in far peripheral vision. Journal of Vision 16, 2 (2016), 10-10.

Giles S Brindley and WS Lewin. 1968. The sensations produced by electrical stimulation of the visual cortex. The fournal of physiology 196, 2 (1968), 479.

A Cowey and ET Rolls. 1974. Human cortical magnification factor and its relation to visual acuity. Experimental Brain Research 21, 5 (1974), 447-454.

Carolina Cruz-Neira, Daniel J Sandin, and Thomas A DeFanti. 1993. Surround-screen projection-based virtual reality: the design and implementation of the CAVE. In Proceedings of the 20th annual conference on Computer graphics and interactive techniques. ACM, 135-142.

Carolina Cruz-Neira, Daniel J Sandin, Thomas A DeFanti, Robert V Kenyon, and John C Hart. 1992. The CAVE: audio visual experience automatic virtual environment. Commun. ACM 35, 6 (1992), 64-73.

PM Daniel and D Whitteridge. 1961. The representation of the visual field on the cerebral cortex in monkeys. The fournal of physiology 159, 2 (1961), 203-221.

Thomas A DeFanti, Gregory Dawe, Daniel J Sandin, Jurgen P Schulze, Peter Otto, Javier Girado, Falko Kuester, Larry Smarr, and Ramesh Rao. 2009. The StarCAVE, a third-generation CAVE and virtual reality OptIPortal. Future Generation Computer Systems 25, 2 (2009), 169-178.

David Dunn, Cary Tippets, Kent Torell, Petr Kellnhofer, Kaan Akşit, Piotr Didyk, Karol Myszkowski, David Luebke, and Henry Fuchs. 2017. Wide Field Of View Varifocal Near-Eye Display Using See-Through Deformable Membrane Mirrors. IEEE Transactions on Visualization and Computer Graphics 23, 4 (2017), 1322-1331.

Steve Grogorick, Michael Stengel, Elmar Eisemann, and Marcus Magnor. 2017. Subtle gaze guidance for immersive environments. In Proceedings of the ACM Symposium on Applied Perception. ACM, 4.

Jonathan C Horton and William F Hoyt. 1991. The representation of the visual field in human striate cortex: a revision of the classic Holmes map. Archives of ophthalmology 109, 6 (1991), 816-824.

Fu-Chung Huang, Kevin Chen, and Gordon Wetzstein. 2015. The light field stereoscope: immersive computer graphics via factored near-eye light field displays with focus cues. ACM Transactions on Graphics (TOG) 34, 4 (2015), 60.

Yoshio Ishiguro and Jun Rekimoto. 2011. Peripheral vision annotation: noninterference information presentation method for mobile augmented reality. In Proceedings of the 2nd Augmented Human International Conference. ACM, 8.

Brett R Jones, Hrvoje Benko, Eyal Ofek, and Andrew D Wilson. 2013a. IllumiRoom: peripheral projected illusions for interactive experiences. In Proceedings of the SIGCHI Conference on Human Factors in Computing Systems. ACM, 869-878.

J Adam Jones, David M Krum, and Mark T Bolas. 2017. Vertical Field-of-View Extension and Walking Characteristics in Head-Worn Virtual Environments. ACM Transactions on Applied Perception (TAP) 14, 2 (2017), 9.

J Adam Jones, J Edward Swan, and Mark Bolas. 2013b. Peripheral stimulation and its effect on perceived spatial scale in virtual environments. IEEE transactions on visualization and computer graphics 19, 4 (2013), 701-710.

Kiyoshi Kiyokawa. 2007. A wide field-of-view head mounted projective display using hyperbolic half-silvered mirrors. In Mixed and Augmented Reality, 2007. ISMAR 2007. 6th IEEE and ACM International Symposium on. IEEE, 207-210.

Paul B Kline and Bob G Witmer. 1996. Distance perception in virtual environments: Effects of field of view and surface texture at near distances. In Proceedings of the Human Factors and Ergonomics Society Annual Meeting, Vol. 40. SAGE Publications Sage CA: Los Angeles, CA, 1112-1116.

Rogier Landman, Jitendra Sharma, Mriganka Sur, and Robert Desimone. 2014. Effect of distracting faces on visual selective attention in the monkey. Proceedings of the National Academy of Sciences 111, 50 (2014), 18037-18042.

Adam M Larson and Lester C Loschky. 2009. The contributions of central versus peripheral vision to scene gist recognition. Fournal of Vision 9, 10 (2009), 6-6.

Jonas Larsson and David J Heeger. 2006. Two retinotopic visual areas in human lateral occipital cortex. The fournal of Neuroscience 26, 51 (2006), 13128-13142.

Lester Loschky, Muriel Boucart, Sebastien Szaffarczyk, Clement Beugnet, Alicia Johnson, and Jia Li Tang. 2015. The contributions of central and peripheral vision 


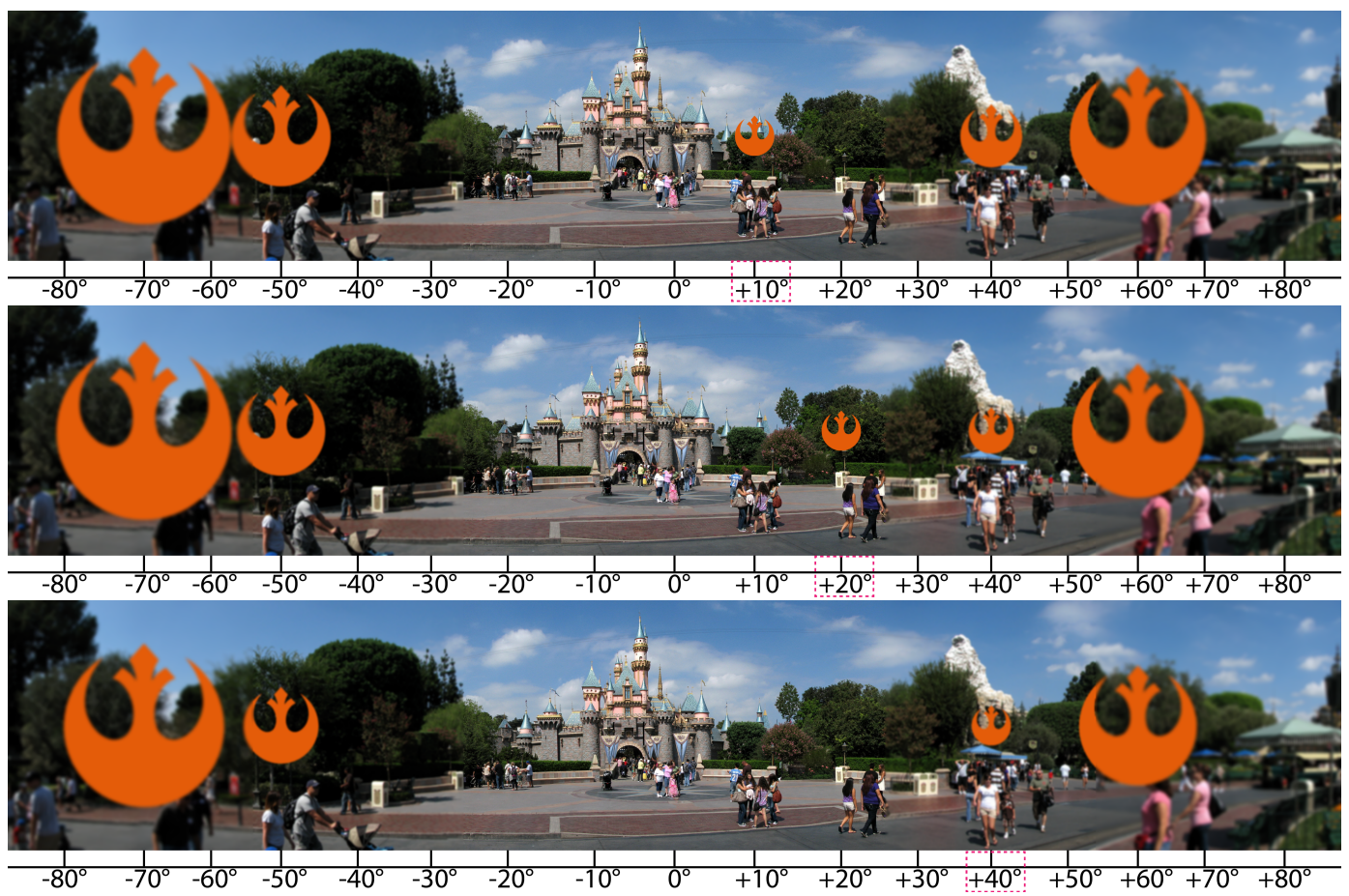

Figure 9: Visualization of equal-efficiency scales for a notional theme park navigation. The Star Wars attraction logo is scaled at various eccentricities using equal-efficiency scales. The target perception times are the perception time of a target pattern of size $5^{\circ}$, at eccentricities (top) $10^{\circ}$, (middle) $20^{\circ}$, and (bottom) $40^{\circ}$ (these are shown with red dashed boxes). We do not use patterns at eccentricity $0^{\circ}$ as their perception time is too fast to be ever matched by patterns at higher eccentricities (regardless of their scaling). Theme park image reproduced with permission of Bernie Kelm, https://www.rocket9.net.

to scene gist recognition with a $180^{\circ}$ visual field. Fournal of vision 15,12 (2015), 570-570.

Andrew Maimone, Andreas Georgiou, and Joel S Kollin. 2017. Holographic near-eye displays for virtual and augmented reality. ACM Transactions on Graphics (TOG) 36, 4 (2017), 85.

Andrew Maimone, Douglas Lanman, Kishore Rathinavel, Kurtis Keller, David Luebke, and Henry Fuchs. 2014. Pinlight displays: wide field of view augmented reality eyeglasses using defocused point light sources. In ACM SIGGRAPH 2014 Emerging Technologies. ACM, 20

Robert W Massof, Lawrence G Brown, Marc D Shapiro, G David Barnett, Frank H Baker and Fuminobu Kurosawa. 2003. 37.1: Invited Paper: Full-Field High-Resolution Binocular HMD. In SID Symposium Digest of Technical Papers, Vol. 34. Wiley Online Library, 1145-1147.

Hajime Nagahara, Yasushi Yagi, and Masahiko Yachida. 2003. Super wide viewer using catadioptrical optics. In Proceedings of the ACM symposium on Virtual reality software and technology. ACM, 169-175.

Toshikazu Ohshima, Hiroyuki Yamamoto, and Hideyuki Tamura. 1996. Gaze-directed adaptive rendering for interacting with virtual space. In Virtual reality annual international symposium, 1996., Proceedings of the IEEE 1996. IEEE, 103-110.

Ismo Rakkolainen, Matthew Turk, and Tobias Höllerer. 2016a. A compact, wide-FOV optical design for head-mounted displays. In Proceedings of the 22nd ACM Conference on Virtual Reality Software and Technology. ACM, 293-294.

I Rakkolainen, M Turk, and T Höllerer. 2016b. A Superwide-FOV Optical Design for Head-Mounted Displays. (2016).

Donghao Ren, Tibor Goldschwendt, YunSuk Chang, and Tobias Höllerer. 2016. Evaluating wide-field-of-view augmented reality with mixed reality simulation. In Virtual Reality (VR), 2016 IEEE. IEEE, 93-102.

George Robertson, Mary Czerwinski, and Maarten Van Dantzich. 1997. Immersion in desktop virtual reality. In Proceedings of the 10th annual ACM symposium on User interface software and technology. ACM, 11-19.

J Rovamo and V Virsu. 1979. An estimation and application of the human cortical magnification factor. Experimental brain research 37, 3 (1979), 495-510.

Mark M Schira, Alex R Wade, and Christopher W Tyler. 2007. Two-dimensional mapping of the central and parafoveal visual field to human visual cortex. Fournal of Neurophysiology 97, 6 (2007), 4284-4295.

Eric L Schwartz. 1980. Computational anatomy and functional architecture of striate cortex: a spatial mapping approach to perceptual coding. Vision research 20, 8 (1980), 645-669.

Liang Shi, Fu-Chung Huang, Ward Lopes, Wojciech Matusik, and David Luebke. 2017. Near-eye light field holographic rendering with spherical waves for wide field of view interactive 3D computer graphics. ACM Transactions on Graphics (TOG) 36, 6 (2017), 236.

Michael J Simpson. 2017. mini-review: Far peripheral vision. Vision research 140 (2017), 96-105.

Hans Strasburger. 2001. Invariance of the psychometric function for character recognition across the visual field. Attention, Perception, \& Psychophysics 63, 8 (2001), 1356-1376.

Hans Strasburger and Maka Malania. 2013. Source confusion is a major cause of crowding. Journal of Vision 13, 1 (2013), 24-24.

Hans Strasburger, Ingo Rentschler, and Martin Jüttner. 2011. Peripheral vision and pattern recognition: A review. Fournal of vision 11, 5 (2011), 13-13.

Simon J Thorpe, Karl R Gegenfurtner, Michèle Fabre-Thorpe, and Heinrich H BuĖlthoff. 2001. Detection of animals in natural images using far peripheral vision. European fournal of Neuroscience 14, 5 (2001), 869-876.

DJ Tolhurst and L Ling. 1987. Magnification factors and the organization of the human striate cortex. Human neurobiology 6, 4 (1987), 247-254.

Christopher W Tyler. 1999. Human symmetry detection exhibits reverse eccentricity scaling. Visual Neuroscience 16, 05 (1999), 919-922.

Mark Weiser and John Seely Brown. 1996. Designing calm technology. PowerGrid fournal 1, 1 (1996), 75-85.

Robert Xiao and Hrvoje Benko. 2016. Augmenting the Field-of-View of Head-Mounted Displays with Sparse Peripheral Displays. In Proceedings of the 2016 CHI Conference on Human Factors in Computing Systems. ACM, 1221-1232.

David J Yates and Tom Stafford. 2011. Insights into the Function and Mechanism of Saccadic Decision Making From Targets Scaled By an Estimate of the Cortical Magnification Factor. Cognitive Computation 3, 1 (2011), 89-93. 